\title{
Quantum nature of Gaussian discord: Experimental evidence and role of system-environment correlations
}

\author{
Vanessa Chille, ${ }^{1}$ Niall Quinn, ${ }^{2}$ Christian Peuntinger, ${ }^{1}$ Callum Croal, ${ }^{2}$ Ladislav Mišta, Jr., ${ }^{3}$ Christoph Marquardt, ${ }^{1}$ \\ Gerd Leuchs, ${ }^{1}$ and Natalia Korolkova ${ }^{2}$ \\ ${ }^{1}$ Max Planck Institute for the Science of Light, Günther-Scharowsky-Straße 1/Bldg. 24, Erlangen, Germany \\ and Institute of Optics, Information and Photonics, University of Erlangen-Nuremberg, Staudtstraße 7/B2, Erlangen, Germany \\ ${ }^{2}$ School of Physics and Astronomy, University of St. Andrews, North Haugh, St. Andrews KY16 9SS, United Kingdom \\ ${ }^{3}$ Department of Optics, Palacký University, 17. listopadu 12, 77146 Olomouc, Czech Republic
}

(Received 2 December 2014; published 13 May 2015)

\begin{abstract}
We provide experimental evidence of quantum features in bipartite states classified as entirely classical according to a conventional criterion based on the Glauber $\mathcal{P}$ function but possessing nonzero Gaussian quantum discord. Their quantum nature is experimentally revealed by acting locally on one part of the discordant state. We experimentally verify and investigate the effect of discord increase under the action of local loss and link it to the entanglement with the environment. Adding an environmental system purifying the state, we unveil the flow of quantum correlations within a global pure system using the Koashi-Winter inequality. For a discordant state generated by splitting a state in which the initial squeezing is destroyed by random displacements, we demonstrate the recovery of entanglement highlighting the role of system-environment correlations.
\end{abstract}

DOI: 10.1103/PhysRevA.91.050301

PACS number(s): 03.67.Hk, 03.67.Bg, 03.65.Yz, 03.67.Mn

As quantum information science develops towards quantum information technology, the question of the efficient use and optimization of resources becomes a burning issue. So far, quantum information processing (QIP) has been mostly thought of as entanglement-enabled technology. Quantum cryptography is an exception, but even there the so-called effective entanglement between the parties plays a decisive role [1,2]. With the advent of new quantum computation paradigms [3] interest in more generic and even nonentangled QIP resources has emerged [4]. Unlike entanglement, the new resources, commonly dubbed as quantum correlations, reside in all states which do not diagonalize in any local product basis. Entanglement and quantum correlations are equivalent notions only for pure states. Quantumness of correlations in separable states is fundamentally related to the noncommutativity of observables, nonorthogonality of states, and properties of quantum measurements, whereas entanglement can be seen as a consequence of the quantum superposition principle. Correlated mixed states are a lucid illustration of the fact that the quantum-classical divide is actually purpose-oriented and that such states, long considered unsuitable for QIP, may become a robust and efficient quantum tool.

In what follows, we will use quantum discord [5] for quantification of quantum correlations. For two systems $A$ and $B$, quantum discord is defined as the difference,

$$
\mathcal{D}^{\leftarrow}(A B)=\mathcal{I}(A B)-\mathcal{J}^{\leftarrow}(A B)
$$

between quantum mutual information $\mathcal{I}(A B)=\mathcal{S}(A)+$ $\mathcal{S}(B)-\mathcal{S}(A B)$ encompassing all correlations present in the system, and the one-way classical correlation $\mathcal{J}^{\leftarrow}(A B)=$ $\mathcal{S}(A)-\inf _{\left\{\hat{\Pi}_{i}\right\}} \mathcal{H}_{\left\{\hat{\Pi}_{i}\right\}}(A \mid B)$, which is operationally related to the amount of perfect classical correlations which can be extracted from the system [6]. Here, $\mathcal{S}$ is the von Neumann entropy of the respective state, $\mathcal{H}_{\left\{\hat{\Pi}_{i}\right\}}(A \mid B)$ is the conditional entropy with measurement on $B$, and the infimum is taken over all possible measurements $\left\{\hat{\Pi}_{i}\right\}$.
In this Rapid Communication, we focus on bipartite mixed Gaussian states relevant in the context of continuousvariable quantum information [7]. The respective correlation quantifier is then Gaussian quantum discord [8,9] defined by Eq. (1), where the minimization in $\mathcal{J}^{\leftarrow}(A B)$ is restricted to Gaussian measurements. The Gaussian discord coincides with unrestricted discord (1) for some states considered by us [10], which confirms the relevance of its use. All nonproduct bipartite Gaussian states have been shown to have nonzero Gaussian discord $[8,11]$ but many of them are termed classical according to the conventional nonclassicality criterion. That is, their density matrix $\hat{\rho}$ can be represented as a statistical mixture of two-mode coherent states $|\alpha\rangle|\beta\rangle$ with well behaved $\mathcal{P}$ function, $\hat{\rho}=\iint_{\mathbb{C}} \mathcal{P}(\alpha, \beta)|\alpha\rangle\langle\alpha|\otimes| \beta\rangle\langle\beta| d^{2} \alpha d^{2} \beta \quad$ [12]. Thus a wide range of states, normally perceived as classical, according to the Gaussian discord should be classified as quantum. Recurring examples of nonzero Gaussian discord in such seemingly classical states raised doubts whether it is a legitimate measure. This apparent discrepancy was discussed in [13]: the nonclassicality criteria can differ in the quantum-optical realm and in information theory. Therefore states classified as quantum in one context, can appear classical in the other. We provide experimental and theoretical evidence that the quantum nature of the bipartite mixed separable states is correctly captured by nonzero Gaussian discord and this quantumness can be revealed by acting merely locally on one part of the state.

Gaussian states are quantum states of systems in infinitely dimensional Hilbert space, e.g., light modes, which possess a Gaussian-shaped Wigner function. Correlations carried by a Gaussian state of two modes $A$ and $B$ are thus completely characterized by the covariance matrix (CM) $\gamma$ [14] with elements $\gamma_{i j}=\left\langle\hat{\xi}_{i} \hat{\xi}_{j}+\hat{\xi}_{j} \hat{\xi}_{i}\right\rangle-2\left\langle\hat{\xi}_{i}\right\rangle\left\langle\hat{\xi}_{j}\right\rangle$, where $\hat{\xi}=\left(\hat{x}_{A}, \hat{p}_{A}, \hat{x}_{B}, \hat{p}_{B}\right)$ is the vector of quadratures. A Gaussian state with CM $\gamma$ is separable if and only if $\gamma^{\left(T_{A}\right)}+i \Omega \geqslant 0$ [15], where $\gamma^{\left(T_{A}\right)}=L \gamma L^{T}$ with $L=\operatorname{diag}(1,-1,1,1)$ and $\Omega=\oplus_{j=1}^{2} i \sigma_{y}$, where $\sigma_{y}$ is the Pauli- $y$ matrix. Gaussian discord carried by the state can be determined from $\gamma$ using the analytic formula derived in [9]. 


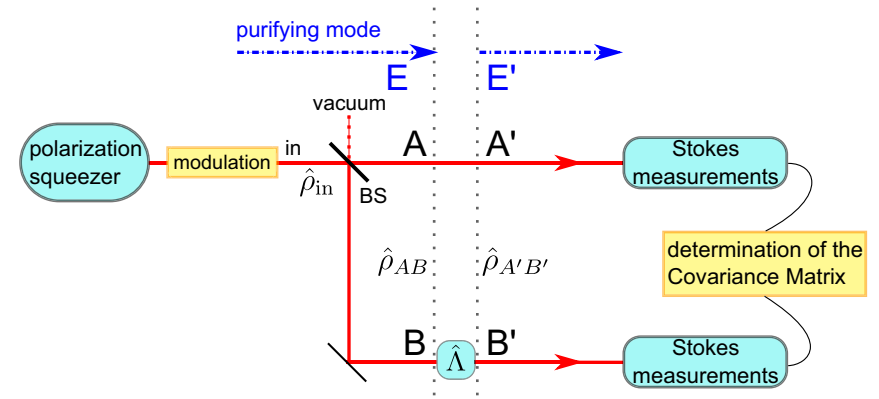

FIG. 1. (Color online) Experimental scheme. BS: beam splitter; $\hat{\rho}_{\text {in }}$ : input state prepared by Gaussian-distributed modulation of coherent or squeezed states; $E\left(E^{\prime}\right)$ : environmental mode purifying mixed state $\hat{\rho}_{A B}\left(\hat{\rho}_{A^{\prime} B^{\prime}}\right) ; \hat{\Lambda}$ : local operation on $B$.

Discord increase under local loss. We prepare a coherent or squeezed optical mode and add noise in the form of Gaussiandistributed random displacements of the $x$ quadrature. The optical mode is then in a classical state given by a convex mixture of coherent states and is split up on a beam splitter (BS) as depicted in Fig. 1. The output two-mode state after the BS with $\mathrm{CM} \gamma_{A B}^{\mathrm{coh}}\left(\gamma_{A B}^{\mathrm{sq}}\right)$ has a nonzero Gaussian discord despite being classical according to the $\mathcal{P}$-function criterion. These quantum states exhibit notable robustness against noise and coupling to the environment. Indeed, as was first shown theoretically for qubits [16-18], quantum correlations can even emerge from a purely classically correlated state under the action of a local noise. This work was then extended to Gaussian states [19], and discord increase under local loss has been experimentally observed for the first time in Ref. [20]. Here we provide a much deeper insight into the exact discord dynamics and offer an elegant intuitive explanation of the effect. First, as we discuss in this Rapid Communication, the observed strong rise in discord was a result of an interesting combination of mere linear loss (an immediate equivalent to the effect discussed in theory papers [16-19]) and some more complicated noise stemming from the detection system. Secondly, and more importantly, we link the discord dynamics to the propagation of quantum correlations in a global system using the Koashi-Winter inequality. We thus pinpoint the role of system-environment correlations and the way that the flow of correlations to the environment affects the system. In this Rapid Communication, we unveil, experimentally and in theory, how the environment can contribute to the manifestation of quantum properties.

Our experimental scheme is shown in Fig. 1. The coherent mode utilized in our experiments stems directly from a femtosecond laser. The used squeezed state is implemented as a polarization squeezed beam generated by the nonlinear Kerr effect of a polarization maintaining fiber [21-23]. For practical reasons the quantum states are encoded in polarization variables. Using intense light fields the Stokes observable in the dark plane $\hat{S}_{\theta}$ is associated with $\hat{x}$ and $\hat{S}_{\theta+\pi / 2}$ with $\hat{p}$ [22]. The squeezed Stokes observable is modulated by an electro-optical modulator (EOM) at the sideband frequency of 18.2 MHz. This is equivalent to a displacement in the dark plane of the quantum states defined at this sideband frequency. Then the mode is divided on a symmetric beam splitter. The

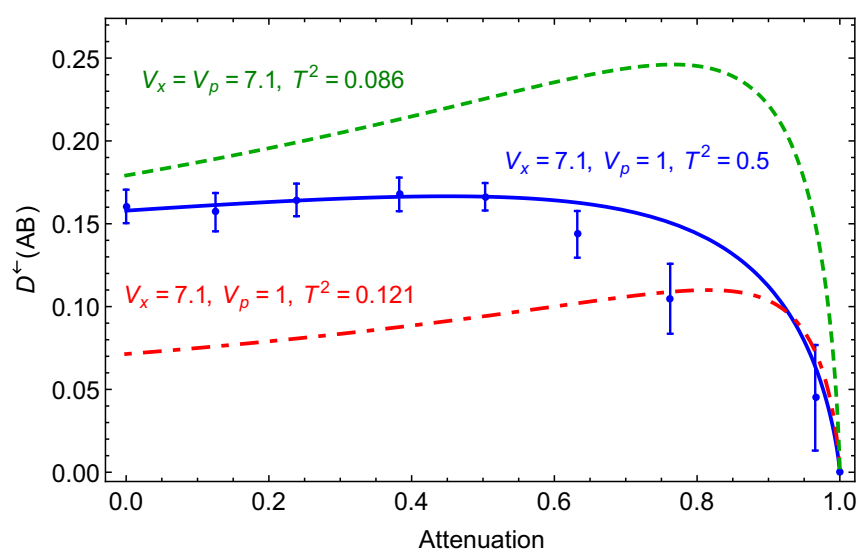

FIG. 2. (Color online) Quantum discord $\mathcal{D}^{\leftarrow}\left(\hat{\rho}_{A B}\right)$ versus attenuation in mode $B$ for modulated coherent state. Theory curve (solid blue) and experiment (blue dots) for modulation in $x$ quadrature, $V_{x}=7.1, V_{p}=1$, and $T^{2}=0.5$. Theory curves for the same input state and $T^{2}=0.121$ (red dot-dashed) and for modulation in both quadratures, $V_{x}=V_{p}=7.1$ and $T^{2}=0.086$ (green dashed).

modes are detected by Stokes measurements and the signal is down-converted at the modulated sideband frequency. The data taken for different displacements is combined computationally to prepare a Gaussian mixed two-mode state. The modulation patterns are chosen such that the initial squeezing is destroyed and the state $\hat{\rho}_{A B}$ is separable. The Stokes measurements allow the determination of its complete CM.

We also provide here a new insight into the interpretation of where the quantumness of correlations comes from. Since quantum discord is related to the noncommutativity of observables, it is often expected that modulation in both conjugate quadratures is required to see quantum behavior. In contrast to all previous discord experiments [20,24-26], to generate discord we modulate the input coherent state only in one of the conjugate quadratures, $\hat{x}_{\text {in }}$, keeping $\hat{p}_{\text {in }}$ at the coherent-state level, $V_{p}=2\left\langle\hat{p}_{\text {in }}^{2}\right\rangle=1$, where $\hat{x}_{\text {in }}\left(\hat{p}_{\text {in }}\right)$ is the $x(p)$ quadrature of mode "in." The local loss is realized by variable attenuation in mode $B$ denoted as $\hat{\Lambda}$ in Fig. 1. Gaussian states with $\mathrm{CM} \gamma_{A B}^{\text {coh }}$ are convex mixtures of nonorthogonal overcomplete coherent basis states. The impossibility to deterministically discriminate between nonorthogonal states is a seminal example of quantumness in separable bipartite states. Thus, intuitively the discord growth under the action of local loss can be attributed to these nonorthogonal basis states becoming less distinguishable with attenuation, although it is difficult to reduce the mechanism behind this effect to a simple single phenomenon [16-18].

Overall, we use and compare two main noise models: linear loss, as described in recent literature, and linear loss plus additional uncorrelated noise, and observe the following discord dynamics depicted in Figs. 2 and 3. The highest discord in $\gamma_{A^{\prime} B^{\prime}}^{\text {coh }}$ is achieved when the initial mode is split on a symmetric BS. Up to a certain attenuation level, $\mathcal{D}^{\leftarrow}\left(\hat{\rho}_{A B}\right)$ grows monotonically with increasing modulation depth, i.e., with $V_{x}=2\left\langle\hat{x}_{\text {in }}^{2}\right\rangle$, and finally drops sharply. The discord rises only very slowly (Fig. 2, blue dots and solid) as, in addition to its positive role, attenuation renders the $\mathrm{CM} \gamma_{A^{\prime} B^{\prime}}^{\text {coh }}$ increasingly asymmetric regarding $A^{\prime}$ and $B^{\prime}$, which suppresses the discord 


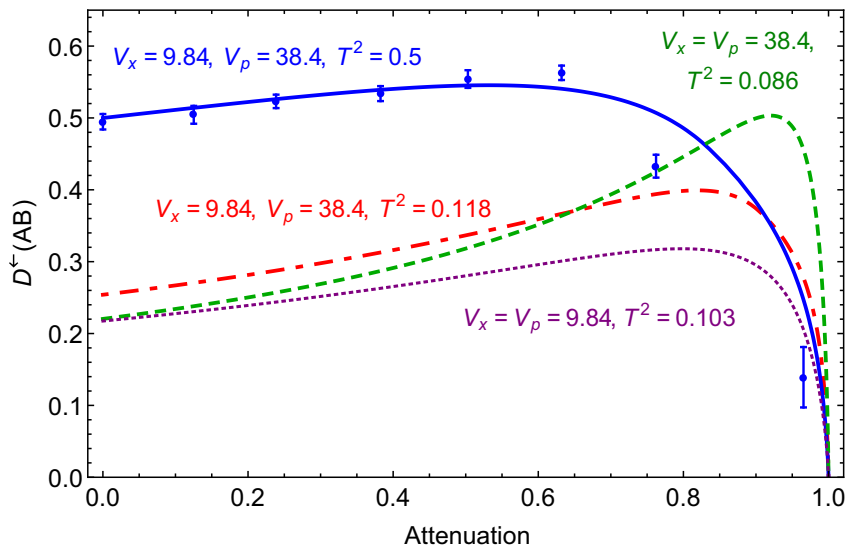

FIG. 3. (Color online) Quantum discord versus attenuation in mode $B$ for modulated squeezed state. Theory curve (blue solid) and experiment (blue dots) for modulation in $x$ quadrature, $V_{x}=9.84$, $V_{p}=38.4$, and $T^{2}=0.5$. Theory curves: for the same input and $T^{2}=0.118$ (red dot-dashed); for $V_{x}=V_{p}=38.4$ and $T^{2}=0.086$ (green dashed); for $V_{x}=V_{p}=9.84$ and $T^{2}=0.103$ (purple dotted).

growth. The gradient in discord can be substantially increased by using an asymmetric BS, such that most of the input beam is reflected into the attenuated mode $B$ (Fig. 2, red dot-dashed). One can obtain the same effect by using the symmetric BS and adding asymmetric noise to the $\mathrm{CM} \gamma_{A^{\prime} B^{\prime}}^{\text {coh }}$, which reflects a limited balancing of the homodyne detectors [20].

Although the quantum effects are observed already when a single quadrature is modulated, Fig. 2 (green dashed) shows that there is an obvious advantage in value and gradient of discord for the input state equally modulated in both quadratures and the asymmetric BS. Incidentally, these dynamics correspond to the measurement results presented in [20], where the additional "noise" (imitating scenario with an asymmetric BS) stems from the imperfections in the detection system.

To get good agreement of theory and experiment (blue dots and solid in Fig. 2) we had to include imperfect common mode rejection (CMR) in homodyne detection into our model. Similar to [20] we model the imperfection by addition of an uncorrelated noise in modes $A^{\prime}$ and $B^{\prime}$ which decreases linearly with attenuation in mode $B^{\prime}$. An even better fit can be achieved without the additional noise, only by using a highly asymmetric BS.

There are several important messages here. First, the largest effect of quantum discord increase under local loss is obtained when the output state is symmetrized with respect to quantum uncertainties in modes $A^{\prime}$ and $B^{\prime}$. In our case this is achieved by using the asymmetric BS, with the optimal ratio determined by the form of the "in" CM (cf. red dot-dashed and blue solid theory curves, Fig. 2). Notably, losses can be turned into a positive control mechanism when using discord as a resource. For example, in the case of imperfect CMR modeled by the asymmetric BS, initial discord is lower (red dot-dashed), which, however, can be counteracted by including attenuation in $B$ so that this additional loss closes the gap between the discord values for the asymmetric and symmetric BS. The effect is even more pronounced for the initial state symmetrically modulated in both quadratures (green dashed), and enhances further when the modulation gets higher (Fig. 3, cf. green-dashed and blue solid curves). Finally, modulation in both incompatible observables is advantageous but not always a prerequisite.

It is interesting to explore whether using a quantum resource initially can bring an advantage. In contrast to [20], the input mixed state in Fig. 3 is created by displacing a squeezed state with approximately $-3 \mathrm{~dB}$ squeezing. Although we still displace the state only along the $x$ axis, it is naturally blurred also in $p$ quadrature due to the antisqueezing and the additional phase noise coming from the propagation in the fiber. This gives an extremely large $p$-quadrature variance, $V_{p}=38.4$. For the discord increase, the only advantage is through these large input variances, irrespective of the quantumness initially present (Fig. 3). However, this initial quantumness does carry a potential to enrich the resultant discordant state. For example, entanglement which would emerge after the BS if no displacement is performed, can still be recovered and used, as we show in the last section.

System-environment correlations provide another control mechanism when using correlated mixed states and give a deeper insight into the quantum effects related to nonzero discord. Assume there is a third mode $E$ carrying maximum information about the state $\hat{\rho}_{A B}$, that might be imprinted onto the environment (Fig. 1). The global state of the system is then the purification $|\psi\rangle_{A B E}$ of $\hat{\rho}_{A B}, \operatorname{Tr}_{E}\left(|\psi\rangle_{A B E}\langle\psi|\right)=\hat{\rho}_{A B}$. The initial purification before the BS is a locally squeezed twomode squeezed vacuum state $|\psi\rangle_{A E}$. Note that the purification for any discordant state is entangled across the $E-(A B)$ splitting, which already links discord and entanglement with the environment. To further analyze the flow of correlations in a global system $|\psi\rangle_{A B E}$, we apply the Koashi-Winter relation [27]

$$
\mathcal{S}(A)=\mathcal{E}_{F}(A E)+\mathcal{J}^{\leftarrow}(A B),
$$

which connects the marginal entropy $\mathcal{S}(A)$, entanglement of formation $(\mathrm{EOF}) \mathcal{E}_{F}(A E)$, and one-way classical correlation $\mathcal{J}^{\leftarrow}(A B)$. The latter is directly linked to discord (1).

In our scheme (Fig. 1), both mutual information $\mathcal{I}(A B)$ and classical correlation $\mathcal{J}^{\leftarrow}(A B)$ decrease with attenuation, but at different rates. First, the rate of decrease in $\mathcal{J}^{\leftarrow}\left(A^{\prime} B^{\prime}\right)$ is higher resulting in an overall discord increase which is already unusual, as the "classical" $\mathcal{J}^{\leftarrow}\left(A^{\prime} B^{\prime}\right)$ decreases faster with attenuation than the "quantum" $\mathcal{I}\left(A^{\prime} B^{\prime}\right)$. As the marginal entropy of $A$ remains unchanged under attenuation in mode $B$, for relation (2) to hold for primed variables the decrease in classical correlation $\mathcal{J}^{\leftarrow}\left(A^{\prime} B^{\prime}\right)$ has to be accompanied by an increase in $\mathcal{E}_{F}\left(A^{\prime} E^{\prime}\right)$ between the unmeasured mode $A^{\prime}$ and the environment.

At high attenuation levels, though, mode $B$ is getting essentially absorbed. Mutual information then decreases at a substantially higher rate, overtaking $\mathcal{J}^{\leftarrow}\left(A^{\prime} B^{\prime}\right)$ and leading to discord drop. The discord decrease is supported further by an increasing asymmetry between $A$ and $B$, as discussed earlier. $\mathcal{D}^{\leftarrow}\left(A^{\prime} B^{\prime}\right), \mathcal{I}\left(A^{\prime} B^{\prime}\right)$, and $\mathcal{J}^{\leftarrow}\left(A^{\prime} B^{\prime}\right)$ naturally hit zero as mode $B$ vanishes. At this point, entanglement with the environment becomes maximal, $\mathcal{E}_{F}\left(A^{\prime} E^{\prime}\right)=\mathcal{S}\left(A^{\prime}\right)$. If we eliminated $B$ completely, it would go over into the initial purification $|\psi\rangle_{A E}$, two-mode squeezed vacuum state. Loosely speaking, all of the quantum correlations are now deposited in the environment. 


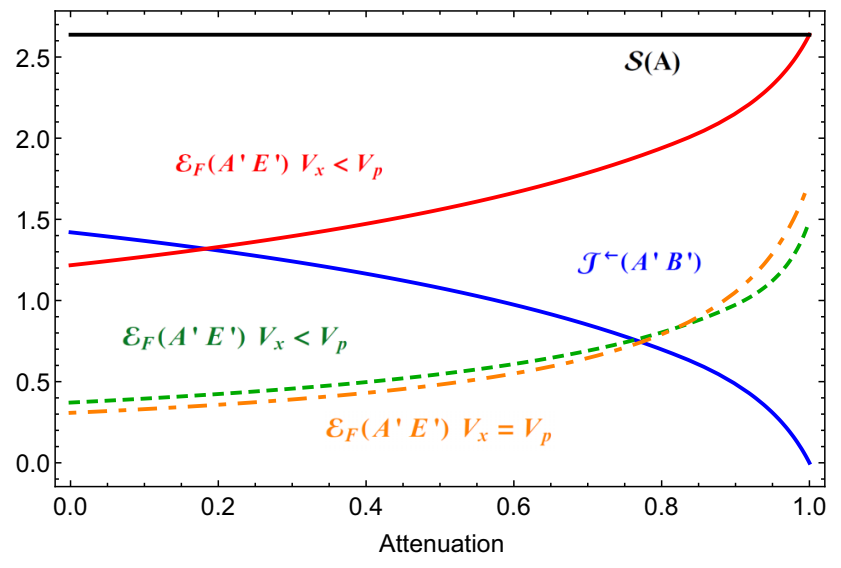

FIG. 4. (Color online) Flow of quantum correlations in the global state $|\psi\rangle_{A B E}$ for the states shown in Fig. 3. Classical correlation (solid blue curve), marginal entropy (solid black line), and systemenvironment EOF for $V_{x}=9.84, V_{p}=38.4$, and $T^{2}=0.5$ (solid red curve). System-environment EOF for same input state and $T^{2}=$ 0.118 (green dashed); for the symmetric input state $V_{x}=V_{p}=38.4$ and $T^{2}=0.086$ (orange dot-dashed).

For computing the EOF of a general Gaussian state $\hat{\rho}_{A E}$ we used the technique of Ref. [28]. As clearly seen in Fig. 4, the growth of discord relates to the increasing EOF with the environment. Figure 4 also witnesses that the Koashi-Winter relation holds for this type of Gaussian states. For the experimentally measured case of Fig. 3, the rising entanglement with environment and decreasing classical correlation between the system modes $A$ and $B$ add up to the constant marginal entropy $\mathcal{S}(A)$. We have also verified that if a measurement is performed on mode $A$, discord always decreases, as does entanglement with the environment $\mathcal{E}_{F}\left(A^{\prime} E^{\prime}\right)$.

In the qubit case, the role of system-environment correlations is particularly eloquent and increase in discord in both cases can be enacted by performing the entangling operation on $A$ and some environmental mode, instead of locally attenuating $B$ [29]. Recently, a further experiment has been proposed linking the open-system dynamics of entanglement to correlations with the environment and discord [30].

Entanglement recovery. The Koashi-Winter relation helped us to trace the exact flow of quantum correlations in an open, global system. Their relevance goes beyond merely theoretical considerations, but can be employed to design communication protocols. As we illustrate below, depending on the precise goal, one can construct multipartite states required for a protocol in question in the following fashion. Discordant states may be combined with some additional mode(s), be it "environmental" mode(s) or auxiliary mode(s). Further, either direct imprinting of some correlated noise via interference between modes, or use of classical information about the preparation of the discordant state can be used to aid generation of the multimode state with desired separability properties, i.e., involving entanglement across certain bipartitions, that might be useful for secret sharing protocols or other security protocols involving several (unequal) parties. Also, direct emulation of an entangled state out of a discordant state is possible. In what follows, we illustrate this idea by one of the simplest protocols possible; another example may be found in [31].
Consider the state $\hat{\rho}_{A B}$ prepared from a state with $-3 \mathrm{~dB}$ of squeezing in $x$ quadrature using Gaussian modulation in the same quadrature. The measured $\mathrm{CM}$ reads

$$
\gamma_{A B}^{\mathrm{sq}}=\left(\begin{array}{cccc}
5.42 & 0.23 & 4.06 & 0.04 \\
0.23 & 19.28 & 0.45 & 17.29 \\
4.06 & 0.45 & 4.73 & 0.55 \\
0.04 & 17.29 & 0.55 & 17.70
\end{array}\right),
$$

where the measurement errors are given in [32]. The local CMs are not squeezed, which verifies that the displacements destroyed the squeezing $[33,34]$. The state of modes $A$ and $B$ is then inevitably separable [35] as witnessed by the non-negativity of the minimal eigenvalue $\min \left\{\operatorname{eig}\left[\left(\gamma_{A B}^{\mathrm{sq}}\right)^{\left(T_{A}\right)}+\right.\right.$ $i \Omega]\}=0.84 \pm 0.02$. However, the state contains quantum correlations as evidenced by $\mathcal{D}^{\leftarrow}(A B)=0.49 \pm 0.01$. The correlations originate from two sources. First, the random displacement $\bar{x}$ of the $x$ quadrature of the input mode "in" yields quantum correlations between separable modes $A$ and $B$ exactly as in the case of the coherent initial state. Secondly, the initial squeezing of mode "in" would alone create entanglement between $A$ and $B$.

Interestingly, there exists a scenario, in which correlations of the system $(A B)$ with a separable environmental mode $\tilde{E}$ allow one to eliminate the displacement noise and recover this entanglement between $A$ and $B$. Note, that mode $\tilde{E}$ is not purifying. Preparation of the state with CM (3) by splitting a randomly displaced squeezed input mode "in" on BS is in fact the preparation of the two-mode reduced state in the entanglement sharing protocol [36]. Imagine that like in the protocol, the $x$ quadrature of $\tilde{E}$ encodes the random displacement $\bar{x}$ as $x_{\tilde{E}}-\bar{x}$. In contrast to the previously considered purifying mode $E$, mode $\tilde{E}$ has been created by local operations and classical communication (LOCC) and hence it is separable from the subsystem. Next, as in [36], mode $B$ is transmitted to the location of mode $\tilde{E}$ where the modes are superimposed on a beam splitter $\mathrm{BS}_{B \tilde{E}}$. As a consequence, the noise caused by the random displacements is partially canceled and the entanglement between modes $A$ and $B$ is restored as desired.

This entanglement recovery reveals two important facts about quantum correlations in the global system $(A B \tilde{E})$. First, it demonstrates that there must exist entanglement across the $A-(B \tilde{E})$ splitting before the beam splitter $\mathrm{BS}_{B \tilde{E}}$ as otherwise it could not create entanglement between modes $A$ and $B$. Second, it is a proof that mode $B$ shares quantum correlations with the subsystem $(A \tilde{E})$ and therefore realizes a true quantum communication between the locations of modes $A$ and $\tilde{E}$, which cannot be replaced by LOCC. Indeed, if mode $B$ was only classically correlated with subsystem $(A \tilde{E})$, it would be possible to replace its transmission by a measurement of its state (which does not disturb the global state), followed by recreation of the state in the location of mode $\tilde{E}$. This is, however, an LOCC operation which cannot establish entanglement across $A-(B \tilde{E})$ splitting.

Instead of physically imprinting a displacement on the third quantum mode $\tilde{E}$ and interfering the mode with mode $B$ on a beam splitter, we have superimposed mode $B$ with vacuum mode $\tilde{E}$ on a beam splitter and implemented equivalent displacement electronically on the measured data. This gives 
us a violation of Duan's separability criterion [23,37,38] $0.91 \pm 0.01<1$, which certifies entanglement between $A$ and $B$.

If we have access to the displacement $\bar{x}$ encoded on mode $\tilde{E}$, entanglement between modes $A$ and $B$ can be recovered by directly performing the reverse displacement on mode $B$ to cancel the modulation. By executing this computationally, we got a violation of Duan's separability criterion of $0.83 \pm$ $0.01<1$ proving that modes $A^{\prime}$ and $B^{\prime}$ after demodulation are entangled.

To place these results in context, consider more involved and counterintuitive experimental protocols, that is, distribution of entanglement by separable states [31,39-41] and entanglement activation from discord [42]. These protocols begin with multipartite discordant but fully separable states. Entanglement then emerges after some local operations on parts of the states. The preparation of the original states involves classical communication between Alice and Bob, which is used to impose correlated noise. In the subsequent stages of the protocols, the noise is removed by interference of the correlated subsystems. The initial discordant correlations are activated into entanglement. The essence of this effect is demonstrated in our Rapid Communication by entanglement recovery using interference with the "environmental mode." Further, we have obtained a better entanglement recovery compared to the quantum interference scenario by imprinting the classical information directly. This features even more clearly the important role of exchange of classical information. We reckon that the same principle is behind the performance of the qubit versions of the discussed protocols but the structure of the communicated classical information can be much more involved in comparison with communication of just a single real number $x$ in our case. In this respect, CVs are unique in unveiling the simple mechanism behind some puzzling effects and lead to a clear intuition how to exploit quantum correlations in separable states.

Acknowledgments. L.M. acknowledges Project No. P205/12/0694 of Czech Science Foundation (GACR). N.K. is grateful for the support provided by the A. von Humboldt Foundation. N.Q. and N.K. acknowledge the support from the Scottish Universities Physics Alliance (SUPA) and the Engineering and Physical Sciences Research Council (EPSRC). The project was supported within the framework of the BMBF grant "QuORep" and in the framework of the International Max Planck Partnership (IMPP) with Scottish Universities.
[1] H. Häseler and N. Lütkenhaus, Phys. Rev. A 81, 060306(R) (2010).

[2] I. Khan, C. Wittmann, N. Jain, N. Killoran, N. Lütkenhaus, Ch. Marquardt, and G. Leuchs, Phys. Rev. A 88, 010302(R) (2013).

[3] E. Knill and R. Laflamme, Phys. Rev. Lett. 81, 5672 (1998).

[4] A. Datta, A. Shaji, and C. M. Caves, Phys. Rev. Lett 100, 050502 (2008).

[5] H. Ollivier and W. H. Zurek, Phys. Rev. Lett. 88, 017901 (2001).

[6] I. Devetak and A. Winter, IEEE Trans. Inf. Theory 50, 3183 (2004).

[7] C. Weedbrook, S. Pirandola, R. Garcia-Patron, N. Cerf, T. Ralph, J. Shapiro, and S. Lloyd, Rev. Mod. Phys. 84, 621 (2012).

[8] G. Adesso and A. Datta, Phys. Rev. Lett. 105, 030501 (2010).

[9] P. Giorda and M. G. A. Paris, Phys. Rev. Lett. 105, 020503 (2010).

[10] S. Pirandola, G. Spedalieri, S. L. Braunstein, N. J. Cerf, and S. Lloyd, Phys. Rev. Lett. 113, 140405 (2014).

[11] L. Mišta, Jr., D. McNulty, and G. Adesso, Phys. Rev. A 90, 022328 (2014).

[12] R. Glauber, Phys. Rev. 131, 2766 (1963); E. C. G. Sudarshan, Phys. Rev. Lett. 10, 277 (1963).

[13] A. Ferraro and M. G. A. Paris, Phys. Rev. Lett. 108, 260403 (2012).

[14] S. L. Braunstein and P. van Loock, Rev. Mod. Phys. 77, 513 (2005).

[15] R. Simon, Phys. Rev. Lett. 84, 2726 (2000).

[16] A. Streltsov, H. Kampermann, and D. Bruß, Phys. Rev. Lett. 107, 170502 (2011).

[17] F. Ciccarello and V. Giovannetti, Phys. Rev. A 85, 010102(R) (2012).
[18] S. Campbell, T. J. G. Apollaro, C. Di Franco, L. Banchi, A. Cuccoli, R. Vaia, F. Plastina, and M. Paternostro, Phys. Rev. A 84, 052316 (2011).

[19] F. Ciccarello and V. Giovannetti, Phys. Rev. A 85, 022108 (2012).

[20] L. S. Madsen, A. Berni, M. Lassen, and U. L. Andersen, Phys. Rev. Lett. 109, 030402 (2012).

[21] N. Korolkova, G. Leuchs, R. Loudon, T. C. Ralph, and C. Silberhorn, Phys. Rev. A 65, 052306 (2002).

[22] J. Heersink, V. Josse, G. Leuchs, and U. L. Andersen, Opt. Lett. 30, 1192 (2005).

[23] R. Dong, J. Heersink, J.-I. Yoshikawa, O. Glöckl, U. L. Andersen, and G. Leuchs, New J. Phys. 9, 410 (2007).

[24] M. Gue, H. M. Chrzanowski, S. M. Assad, T. Symul, K. Modi, T. C. Ralph, V. Vedral, and P. K. Lam, Nat. Phys. 8, 671 (2012).

[25] U. Vogl, R. T. Glasser, Q. Glorieux, J. B. Clark, N. V. Corzo, and P. D. Lett, Phys. Rev. A 87, 010101(R) (2013).

[26] R. Blandino, M. G. Genoni, J. Etesse, M. Barbieri, M. G. A. Paris, P. Grangier, and R. Tualle-Brouri, Phys. Rev. Lett. 109, 180402 (2012).

[27] M. Koashi and A. Winter, Phys. Rev. A 69, 022309 (2004).

[28] M. M. Wolf, G. Giedke, O. Krüger, R. F. Werner, and J. I. Cirac, Phys. Rev. A 69, 052320 (2004); G. Adesso and F. Illuminati, ibid. 72, 032334 (2005).

[29] R. Tatham and N. Korolkova, Phys. Scr., T 160, 014040 (2014).

[30] G. H. Aguilar, O. J. Farias, A. Valdes-Hernandez, P. H. Souto Ribeiro, L. Davidovich, and S. P. Walborn, Phys. Rev. A 89, 022339 (2014).

[31] Ch. Peuntinger, V. Chille, L. Mišta, N. Korolkova, M. Förtsch, J. Korger, Ch. Marquardt, and G. Leuchs, Phys. Rev. Lett. 111, 230506 (2013). 
[32] The error for the CM given in Eq. (3) of the main text was estimated to be

$$
\left(\begin{array}{llll}
0.05 & 0.02 & 0.03 & 0.01 \\
0.02 & 0.17 & 0.01 & 0.15 \\
0.03 & 0.01 & 0.04 & 0.02 \\
0.01 & 0.15 & 0.02 & 0.16
\end{array}\right) .
$$

[33] The CM exhibits a weak global squeezing which is an artefact originating from nonzero $x-p$ correlations caused by limited balancing between the Stokes measurement setups (see also [34]).

[34] T. Eberle, V. Händchen, and R. Schnabel, Opt. Express 21, 11546 (2013).

[35] M. S. Kim, W. Son, V. Bužek, and P. L. Knight, Phys. Rev. A 65, 032323 (2002).
[36] L. Mišta, Jr., Phys. Rev. A 87, 062326 (2013).

[37] L.-M. Duan, G. Giedke, J. I. Cirac, and P. Zoller, Phys. Rev. Lett. 84, 2722 (2000).

[38] V. Giovannetti, S. Mancini, D. Vitali, and P. Tombesi, Phys. Rev. A 67, 022320 (2003).

[39] T. S. Cubitt, F. Verstraete, W. Dür, and J. I. Cirac, Phys. Rev. Lett. 91, 037902 (2003).

[40] C. E. Vollmer, D. Schulze, T. Eberle, V. Händchen, J. Fiurášek, and R. Schnabel, Phys. Rev. Lett. 111, 230505 (2013).

[41] A. Fedrizzi, M. Zuppardo, G. G. Gillett, M. A. Broome, M. de Almeida, M. Paternostro, A. G. White, and T. Paterek, Phys. Rev. Lett. 111, 230504 (2013).

[42] G. Adesso, V. D’Ambrosio, E. Nagali, M. Piani, and F. Sciarrino, Phys. Rev. Lett. 112, 140501 (2014). 